\title{
INTEGRATION THEORY ON INFINITE-DIMENSIONAL MANIFOLDS
}

\author{
BY \\ HUI-HSIUNG KUO( $\left.{ }^{1}\right)$
}

\begin{abstract}
The purpose of this paper is to develop a natural integration theory over a suitable kind of infinite-dimensional manifold. The type of manifold we study is a curved analogue of an abstract Wiener space.

Let $H$ be a real separable Hilbert space, $B$ the completion of $H$ with respect to a measurable norm and $i$ the inclusion map from $H$ into $B$. The triple $(i, H, B)$ is an abstract Wiener space. $B$ carries a family of Wiener measures.

We will define a Riemann-Wiener manifold to be a triple $(\mathscr{W}, \tau, g)$ satisfying specific conditions. $\mathscr{W}$ is a $C^{j}$-differentiable manifold $(j \geqq 3)$ modelled on $B$ and, for each $x$ in $\mathscr{W}, \tau(x)$ is a norm on the tangent space $T_{x}(\mathscr{W})$ of $\mathscr{W}$ at $x$ while $g(x)$ is a densely defined inner product on $T_{x}(\mathscr{W})$.

We show that each tangent space is an abstract Wiener space and there exists a spray on $\mathscr{W}$ associated with $g$. For each point $x$ in $\mathscr{W}$ the exponential map, defined by this spray, is a $C^{j-2}$-homeomorphism from a $\tau(x)$-neighborhood of the origin in $T_{x}(\mathscr{W})$ onto a neighborhood of $x$ in $\mathscr{W}$. We thereby induce from Wiener measures of $T_{x}(\mathscr{W})$ a family of Borel measures $q_{t}(x, \cdot), t>0$, in a neighborhood of $x$. We prove that $q_{t}(x, \cdot)$ and $q_{s}(y, \cdot)$, as measures in their common domain, are equivalent if and only if $t=s$ and $d_{g}(x, y)$ is finite. Otherwise they are mutually singular. Here $d_{g}$ is the almost-metric (in the sense that two points may have infinite distance) on $\mathscr{W}$ determined by $g$. In order to do this we first prove an infinite-dimensional analogue of the Jacobi theorem on transformation of Wiener integrals.
\end{abstract}

0. Introduction. The notion of an abstract Wiener space, first introduced by Gross [8], generalizes that of the classical Wiener space. Wiener [16] put a measure in the Banach space $C$ consisting of the real-valued continuous functions on $[0,1]$ which vanish at 0 with the sup norm. The subset $C^{\prime}$ consisting of the absolutely continuous functions in $C$ with square integrable derivative forms a Hilbert space with the inner product $\langle x, y\rangle=\int_{0}^{1} x^{\prime}(t) y^{\prime}(t) d t$. Let $i$ be the inclusion map from $C^{\prime}$ into $C$; then the triple $\left(i, C^{\prime}, C\right)$ is known as the classical Wiener space.

Received by the editors August 3, 1970.

AMS 1969 subject classifications. Primary 2846, 6005; Secondary 5750, 5755.

Key words and phrases. Integration theory, infinite-dimensional manifold, abstract Wiener space, Wiener measures, Jacobi Theorem, Riemann-Wiener manifold, Riemannian manifold, admissible transformation, spray, exponential map, Radon-Nikodym derivative, equivalenceperpendicularity dichotomy.

(1) Results are included in the author's doctoral dissertation at Cornell University in 1970. The author wishes to express his deep gratitude to Professor Leonard Gross under whose direction the thesis was written. 
Let $L$ be a locally convex real linear space and $L^{*}$ its topological dual space. Let ( , ) denote the natural pairing of $L^{*}$ and $L$. A tame set (also known as a cylinder set) in $L$ is a set $D$ of the form

$$
D=\left\{x \in L ;\left(\left(y_{1}, x\right),\left(y_{2}, x\right), \ldots,\left(y_{n}, x\right)\right) \in E\right\}
$$

where $y_{1}, y_{2}, \ldots, y_{n}$ are in $L^{*}$ and $E$ is a Borel set in $\boldsymbol{R}^{n}$. If $K$ is any finite-dimensional subspace of $L^{*}$ containing $y_{1}, y_{2}, \ldots, y_{n}$, then $D$ is said to be based on $K$. Let $\mathscr{R}$ be the collection of all tame sets in $L$ and $\mathscr{S}_{K}$ the family of sets in $\mathscr{R}$ which are based on $K$. It is easy to see that $\mathscr{R}$ is a ring and $\mathscr{P}_{K}$ is a $\sigma$-ring. A real-valued nonnegative finitely-additive function $\nu$ on $\mathscr{R}$ is called a cylinder set measure on $L$ if $\nu(L)=1$ and $\nu$ is countably additive on each of the $\sigma$-rings $\mathscr{S}_{K}$.

A weak distribution over $L$ is an equivalence class of linear maps $F$ from $L^{*}$ to the space of random variables over a probability space $(\Omega, m)$ (depending on $F$ ). Two such maps $F_{1}$ and $F_{2}$ are equivalent if for any finite set $y_{1}, \ldots, y_{n}$ in $L^{*}$ the joint distribution of $F_{j}\left(y_{1}\right), \ldots, F_{j}\left(y_{n}\right)$ in $\boldsymbol{R}^{n}$ is the same for $j=1$ or 2 . This definition is due to I. E. Segal [14]. It is easy to see that the two concepts-cylinder set measure and weak distribution-are equivalent.

The weak distribution which will be of interest to us is the canonical normal distribution $n_{t}$ on a real separable Hilbert space $H$ with (variance) parameter $t>0$ defined as follows. If $F_{t}$ is a representative of $n_{t}$ then for each element $y$ in $H^{*}$ (which is identified with $H$ ) $F_{t}(y)$ is normally distributed with mean 0 and variance $t|y|^{2}$. Henceforth $H$ will denote a real separable Hilbert space with its norm $|\cdot|$ and inner product $\langle$,$\rangle . It follows that F_{t}$ takes orthogonal vectors into stochastically independent random variables. The cylinder set measure $\mu_{t}$ associated with $n_{t}$ is called Gaussian measure on $H$. A tame set $D$ in $H$ can be written as $D=P^{-1}(E)$, where $P$ is a finite-dimensional orthogonal projection on $H$ and $E$ is a Borel set in $P H$. Then $\mu_{t}$ can be characterized by

$$
\mu_{t}(D)=(2 \pi t)^{-k / 2} \int_{E} e^{-|x|^{2} / 2 t} d x
$$

where $k$ is the dimension of $P H$ and $d x$ is Lebesgue measure in $P H . \mu_{t}$ is not countably additive on $\mathscr{R}$ unless $H$ is finite dimensional.

We denote by $\mathscr{F}$ the directed set of all finite-dimensional projections on $H$ directed by inclusion of the ranges. A tame function on $H$ is a function of the form $f=\varphi \circ P$, where $P \in \mathscr{F}$ and $\varphi$ is a Baire function on $P H$. Each tame function $f$ is associated with a random variable $f$ in the following way. Let $F_{t}$ be a fixed representative of $n_{t}$. Let $y_{1}, \ldots, y_{k}$ be an orthonormal basis for $P H$ then define $f=$ $\varphi\left(F_{t}\left(y_{1}\right), \ldots, F_{t}\left(y_{k}\right)\right)$. $\tilde{f}$ depends only on $f$ and $F_{t}$, and is independent of the expression of $f$ in terms of $\varphi$ and $y_{1}, \ldots, y_{k}$.

A measurable norm on $H$ is a norm $\|x\|$ on $H$ with the property that for every $\varepsilon>0$ there exists $P_{0} \in \mathscr{F}$ such that

$$
\operatorname{Prob}_{1}\left(\|P x\|^{\sim}>\varepsilon\right)<\varepsilon \quad \text { whenever } P \in \mathscr{F} \text { and } P \perp P_{0} .
$$


Here Prob $_{1}$ refers to the probability of the indicated event with respect to the probability measure associated with $n_{1}$.

From now on $\|\cdot\|$ denotes a fixed measurable norm on $H$. The definition of measurable norm implies that there exists a constant $\beta$ such that $\|x\| \leqq \beta|x|$ for all $x$ in $H$. Denote by $B$ the completion of $H$ with respect to $\|\cdot\| . B$ is a real separable Banach space and any real separable Banach space can be obtained (up to a linear isometry) from a Hilbert space in this manner [8]. Let $i$ be the inclusion map from $H$ into $B$ and $j$ the embedding (by restriction) of $B^{*}$ into $H^{*}$. Henceforth we will make the following identifications $H^{*} \equiv H$ and $B^{*} \equiv j B^{*}$. Therefore we have the situation $B^{*} \subset H \subset B$. Since $B^{*}$ separates points of $H, B^{*}$ is dense in $H$. Note that $\|x\| \leqq \beta|x|$ for all $x \in H$ implies that $|x| \leqq \beta\|x\|_{B^{*}}$ for all $x \in B^{*}$. The triple $(i, H, B)$ is called an abstract Wiener space. This definition is due to L. Gross [8].

Let $\left(\right.$, ) denote the natural pairing of $B^{*}$ and $B$. It is a consequence of the above identifications that $\langle x, y\rangle=(x, y)$ whenever $x$ is in $B^{*}$ and $y$ in $H$. Moreover, the elements of $B^{*}$ are precisely those elements $x$ of $H$ having the property that there exists a constant $\alpha$ (depending on $x$ ) such that $|\langle x, y\rangle| \leqq \alpha\|y\|$ for all $y \in H$.

$\mu_{t}$ induces a cylinder set measure $\rho_{t}$ in $B$ as follows: If $y_{1}, \ldots, y_{k}$ are in $B^{*}$ and $E$ is a Borel set in $\boldsymbol{R}^{k}$ define

$$
\rho_{t}\left(\left\{x \in B ;\left(\left(y_{1}, x\right), \ldots,\left(y_{k}, x\right)\right) \in E\right\}\right)=\mu_{t}\left(\left\{h \in H ;\left(\left\langle y_{1}, h\right\rangle, \ldots,\left\langle y_{k}, h\right\rangle\right) \in E\right\}\right) .
$$

It is proved in [8] that $\rho_{t}$ is countably additive on the ring of tame sets of $B$. Therefore $\rho_{t}$ has a unique countably additive extension $p_{t}$ to the $\sigma$-ring generated by all tame sets in $B$. By the separability of $B$ and a simple application of the HahnBanach theorem it can be easily shown that this $\sigma$-ring is exactly the Borel field of $B$. $p_{t}$ is called Wiener measure on $B$ with variance parameter $t$.

It is also proved in [8] that the identity map on $B^{*}$ regarded as a densely defined map of $H$ into random variables over the probability space $\left(B, p_{t}\right)$ extends uniquely to a representative of the normal distribution $n_{t}$.

We will use the following notations. $f_{x}$ denotes the Fréchet derivative of $f$ at $x$. $\mathscr{B}(X, Y)$ denotes the normed linear space of bounded operators from $X$ into $Y$ with operator norm. $\mathscr{B}_{1}(H, H)$ and $\mathscr{B}_{2}(H, H)$ denote the Banach spaces of trace class operators and Hilbert-Schmidt operators of $H$ respectively. $\|\cdot\|_{1}$ and $\|\cdot\|_{2}$ denote the trace class norm and Hilbert-Schmidt norm respectively.

We need the following propositions later on. The proofs are easy and hence omitted.

Proposition 0.1. If $\|\cdot\|$ is $p_{1}$-square integrable, then every continuous operator from $B$ into $B^{*}$ is a trace class operator when it is restricted to $H$. In fact $\left\|\left.A\right|_{H}\right\|_{1}$ $\leqq \int_{B}\|x\|^{2} d p_{1}(x)\|A\|_{B, B^{*}}$ for all $A \in \mathscr{B}\left(B, B^{*}\right)$.

Proposition 0.2. Let $X$ be a real Banach space and $A$ a continuous operator from $X$ into $B$ with image contained in $H$ (in $\left.B^{*}\right)$. Then $A$ is a continuous operator from $X$ into $H$ (into $B^{*}$ ). 
Proposition 0.3. Let $U$ be a convex open set in $B$. Suppose $K$ is a Fréchet differentiable transformation from $U$ into $B$ with image in $H$. Moreover $K_{x}(B) \subset B^{*}$ for all $x$ in $U$ and the transformation $x \rightarrow K_{x}$ from $U$ into $\mathscr{B}\left(B, B^{*}\right)$ is continuous. Let $x_{0}$ be a fixed point in $U$. Then $K x-K x_{0} \in B^{*}$ for all $x \in U$ and the function

$$
x \rightarrow\left(K x-K x_{0}, x\right)
$$

is continuous from $U$ into $\boldsymbol{R}$.

In §I we will define a class of (nonlinear) transformations and prove the Jacobi theorem on transformation of Wiener integrals under such transformations. In §II we will define Riemann-Wiener manifold and construct a spray on it. Then we study the exponential map associated with this spray. In §III we use the exponential map to define local measures $q_{t}(x, \cdot)$ for each point $x$ on this manifold and study their properties.

Our eventual objective is to construct a Brownian motion on a Riemann-Wiener manifold. The techniques we have developed in this paper will, we believe, be essential tools for this purpose. Moreover, the measures $q_{t}(x, \cdot)$ are local first order approximations to the transition probabilities of the Brownian motion. However we shall not study the Brownian motion in this paper.

This work is closely related to results announced by Daleckiĭ and Šnaĭderman [4]. They prove absolute continuity of certain measures on infinite-dimensional manifolds. Their manifolds are closely related to ours. However they study only manifolds modelled on Hilbert space and use a different exponential map from the one we use. The measures whose absolute continuity they study are different from ours.

\section{The Jacobi theorem on transformation of Wiener integrals.}

(a) Statement of the first main result Theorem I.4. Suppose $(i, H, B)$ is an abstract Wiener space with the $H$-norm denoted by $|\cdot|$ and the $B$-norm by $\|\cdot\|$. Assume that the function $\|\cdot\|$ is of class $C^{1}$ off the origin. Also we use $\beta$ to denote a constant such that $\|x\| \leqq \beta|x|$ for all $x \in H$.

Definition I.1. A transformation $T$ from one open subset $U$ of $B$ to another open subset $V$ of $B$ is said to be admissible if it is a homeomorphism from $U$ onto $V$ of class $C^{1}$ and satisfies the following conditions:

(ad-1) $T x-x \in H, T_{x}: B \rightarrow B$ is nonsingular with $\left(T_{x}-I\right)(B) \subset B^{*}$ and

$$
\left.\left(T_{x}-I\right)\right|_{H} \in \mathscr{B}_{1}(H, H) \text { for all } x \in U .
$$

(ad-2) The mapping $x \rightarrow T_{x}-I$ is continuous from $U$ (with the $B$-topology) into $\mathscr{B}\left(B, B^{*}\right)$ (with the uniform topology).

(ad-3) The mapping $\left.x \rightarrow\left(T_{x}-I\right)\right|_{H}$ is continuous from $U$ (with the $B$-topology) into $\mathscr{B}_{1}(H, H)$ (with the trace class norm topology).

Proposition I.2. The inverse of an admissible transformation is also admissible. The composition of two admissible transformations is also admissible. 
Proof. Since the proof is straightforward, we omit it. Q.E.D.

Proposition I.3. Suppose $\|\cdot\|$ is $p_{1}$-square integrable. Let $T$ be a homeomorphism from an open subset $U$ of $B$ onto another open subset $V$ of $B$ of class $C^{1}$. For all $x \in U, T x-x \in H$ and $T_{x}$ is nonsingular from $B$ into itself with $\left(T_{x}-I\right)(B) \subset B^{*}$. Furthermore the map $x \rightarrow T_{x}-I$ is continuous from $U$ into $\mathscr{B}\left(B, B^{*}\right)$. Then $T$ is admissible.

Proof. Obvious by noting Proposition 0.1. Q.E.D.

THEOREM I.4. If $T$ is an admissible transformation from an open subset $U$ of $B$ onto another open subset $V$ of $B$, then $p_{t} \circ T$ and $p_{t}$, as measures on $U$, are equivalent. Moreover the Radon-Nikodym derivative of $p_{t} \circ T$ with respect to $p_{t}$ is given by

$$
\frac{d p_{t} \circ T}{d p_{t}}(x)=g_{t}(x), \quad x \in U,
$$

where $g_{t}(x)=\exp \left\{\left[-2(T x-x, x)-|T x-x|^{2}\right] / 2 t\right\} \operatorname{det}\left|T_{x}\right|$ and $($,$) is the natural$ pairing of $B^{*}$ and $B$.

REMARK (i). $g_{t}$ is defined almost everywhere on $U$ with respect to $p_{s}$ for any $s>0$, in particular w.r.t. $p_{t}$. Furthermore it is Wiener measurable and positive a.e. This can be seen as follows. Let $x_{0} \in U$ then there exists a positive real number $r$ such that $b\left(x_{0},\|\cdot\|, r\right) \equiv\left\{x \in B ;\left\|x-x_{0}\right\|<r\right\} \subset U$. For $x \in b\left(x_{0},\|\cdot\|, r\right)$ we have

$$
g_{t}(x)=\exp \left[\left(K x_{0}, x\right) t^{-1}\right] \exp \left\{\left[-2\left(K x-K x_{0}, x\right)-|K x|^{2}\right] / 2 t\right\} \operatorname{det}\left|T_{x}\right|
$$

where $K x=T x-x$. The assertion follows from Proposition 0.3.

REMARK (ii). The conclusion of Theorem I.4 is equivalent to the following. For any Wiener measurable functional $F$ on $V$ which makes either side of (1.1) exist, then the other side also exists and they are equal:

$$
\int_{V} F(y) d p_{t}(y)=\int_{U} F \circ T(x) g_{t}(x) d p_{t}(x) .
$$

(b) The integral formula for globally defined transformations. In the following we will make no difference between $K_{x}$ and $\left.K_{x}\right|_{H}$ whenever there is no confusion. For examples $\left\|K_{x}\right\|_{1}$ means the trace class norm of $\left.K_{x}\right|_{H}$ and $\left\|K_{x}\right\|_{H, H}$ means the norm of $K_{x}$ as a mapping from $H$ into $H$.

THEOREM I.5. Let $T=I+K$ be a transformation of class $C^{1}$ from $B$ into itself satisfying the following conditions:

(1) $K(B) \subset H$ and $K_{x}(B) \subset B^{*}, K_{x} \in \mathscr{B}_{1}(H, H)$ for all $x \in B$.

(2) The mapping $x \rightarrow K_{x}$ is continuous from $B$ (with the $B$-topology) into $\mathscr{B}\left(B, B^{*}\right)$ (with the uniform topology), also from $B$ into $\mathscr{B}_{1}(H, H)$ (with the trace class norm topology).

(3) There exist constants $0<M<\infty, 0 \leqq c<1$ such that for all $x \in B,\left\|K_{x}\right\|_{1} \leqq M$, $\|K(x)-K(0)\|_{B^{*}} \leqq M,\left\|K_{x}\right\|_{H, H} \leqq c$ and $\left\|K_{x}\right\|_{B, B} \leqq c$. 
Then for any integrable functional $F$ on $B$, we have

$$
\int_{B} F(y) d p_{t}(y)=\int_{B} F \circ T(x) g_{t}(x) d p_{t}(x)
$$

and $p_{t}\left\{g_{t}>0\right\}=1$, where $g_{t}(x)=\exp \left\{\left[-2(K x, x)-|K x|^{2}\right] / 2 t\right\}$ det $\left|T_{x}\right|$.

Proof. Step (i). It is sufficient to prove (1.2) for bounded continuous functions with bounded support. Let $F$ be such a function; we may assume that the support of $F$ is contained in the ball $b(K(0), R,\|\cdot\|)$.

Let $\mathscr{F}^{*}=\left\{P \in \mathscr{F} ; P(H) \subset B^{*}\right\}$. Each $P \in \mathscr{F}^{*}$ can be extended to a continuous projection $Q$ of $B$ in the following way: Suppose $P(x)=\left\langle x, e_{1}\right\rangle e_{1}+\cdots+\left\langle x, e_{n}\right\rangle e_{n}$, $x \in H, e_{1} \cdots e_{n} \in B^{*}$, then define

$$
Q(x)=\left(x, e_{1}\right) e_{1}+\cdots+\left(x, e_{n}\right) e_{n}, \quad x \in B .
$$

For the rest of the proof, $P$ and $Q$ are related in the above way. By Corollary 5.5 of [7], Corollary 3 of [8] and elementary measure theory, we have

$$
\int_{B} F(y) d p_{t}(y)=\lim _{P \rightarrow I \text { through } \mathscr{F} \cdot} \int_{P H} F(y) d \mu_{t}(y) .
$$

We may take $\{P\}$ to be a sequence converging to $I$ strongly through $\mathscr{F} *$. Let $T_{P}=I+P K P$, then $T_{P}$ is a differentiable homeomorphism from $P H$ onto itself, and the Jacobian theorem for finite-dimensional space gives

$$
\begin{aligned}
\int_{P H} F(y) d \mu_{t}(y) & =\int_{P H} F \circ T_{P}(x)\left(g_{t}\right)_{P}(x) d \mu_{t}(x) \\
& =\int_{P H} F \circ T_{P}(P x)\left(g_{t}\right)_{P}(x) d \mu_{t}(x)
\end{aligned}
$$

where $\left(g_{t}\right)_{P}(x)=\exp \left\{\left[-2(P K P x, x)-|P K P x|^{2}\right] / 2 t\right\} \operatorname{det}\left|I+P K_{P x} P\right|$. But $G_{P}(x)=$ $F \circ T_{P}(P x)\left(g_{t}\right)_{P}(x)$ is a tame function based on $P H$, hence

$$
\int_{P H} G_{P}(x) d \mu_{t}(x)=\int_{B} G_{\tilde{P}}(x) d p_{t}(x) .
$$

Define a function $J_{Q}: B \rightarrow R$ by

$$
\begin{aligned}
J_{Q}(x)=F \circ(I+ & P K P) \\
& \circ Q(x) \exp \left\{\left[-2(P K Q x, x)-|P K Q x|^{2}\right] / 2 t\right\} \operatorname{det}\left|I+P K_{Q x} P\right|,
\end{aligned}
$$

then, obviously, $J_{Q}$ is a continuous functional on $B$ and its restriction on $H$ is $G_{P}$ since $Q x=P x$ for all $x$ in $H$ by the definition of $Q$. Therefore $G_{P}=J_{Q}$ almost everywhere by Corollary 3 of [8]. It follows that

$$
\int_{B} G_{\tilde{p}}(x) d p_{t}(x)=\int_{B} J_{Q}(x) d p_{t}(x)
$$


Combining (1.3)-(1.7), we have

$$
\int_{B} F(y) d p_{t}(y)=\lim _{P \rightarrow I \text { through } \mathscr{F} \cdot} \int_{B} J_{Q}(x) d p_{t}(x) .
$$

Step (ii). We prove that there exists a subsequence $P$ such that $F \circ(I+P K P) \circ Q$ converges almost everywhere to $F \circ T$ as $P \rightarrow I$ through $\mathscr{F} *$.

First we note that the inclusion maps $i: H \rightarrow B$ and $j: B^{*} \rightarrow H$ are compact. Therefore bounded subsets in $B^{*}$ are precompact in $H$. So the set

$$
\left\{x \in B^{*} ;\|x\|_{B^{*}} \leqq M\right\}
$$

is precompact in $H$.

Let $S$ be any compact subset of $H$, then $(I-P) x \rightarrow 0$ as $P \rightarrow I$ through $\mathscr{F} *$ uniformly in $S$. But $K(B) \subset K(0)+\left\{x \in B^{*} ;\|x\|_{B^{*}} \leqq M\right\}$ by assumption, whence $(I-P) K Q(x) \stackrel{H}{\rightarrow} 0$ uniformly for $x \in B$ as $P \rightarrow I$ through $\mathscr{F} *$, where $\stackrel{H}{\rightarrow}$ means in the $H$-topology. $H$-topology is stronger than the $B$-topology, so $(I-P) K Q(x) \stackrel{B}{\rightarrow} 0$ uniformly for $x \in B$ as $P \rightarrow I$ through $\mathscr{F}^{*}$. $F$ is a continuous functional in $B$, therefore

$$
\lim _{P \rightarrow I \text { through } \mathscr{F}^{*}} F \circ(I+P K P) \circ Q=\lim _{P \rightarrow I \text { through } \mathscr{F} \cdot} F \circ(I+K) \circ Q
$$

everywhere in $B$. On the other hand since $F \circ(I+K)$ is continuous in $B$, there exists a subsequence of $P$, still denoted by $P$, such that $F \circ(I+K) \circ Q \rightarrow F \circ(I+K)$ $=F \circ T$ a.e. in $B$ as $P \rightarrow I$ through $\mathscr{F}^{*}$. Therefore $F \circ(I+P K P) \circ Q \rightarrow F \circ T$ a.e. in $B$.

Step (iii). Let $\pi(x)=\Delta\left(K_{x}\right)=\operatorname{det}\left|I+K_{x}\right|$, then the condition (2) and Lemma 4.1 of [9] imply that $\pi$ is a continuous function on $B$. Furthermore the condition (3) implies that $K_{x} \in \mathscr{S}$ (the notation used in [9]) for all $x \in B$.

By Lemma 4.1 of [9] there exist two positive finite numbers $a, b$ (which depend only on $M$ and $c$ such that $a<\pi(x)<b$. Let $\pi_{P}(x)=\operatorname{det}\left|I+P K_{Q K} P\right|$. We know that $\pi \circ Q \rightarrow \pi$ in probability, so there exists a subsequence, still denoted by $P$, such that $\pi \circ Q \rightarrow \pi$ almost everywhere. Therefore, using Corollary 3.2 of [7], it follows that $\pi_{P} \rightarrow \pi$ a.e.

Step (iv). Let $\sigma_{P}(x)=F \circ(I+P K P) \circ Q(x) \exp \{-(K Q x, Q x) / t\}$. By writing $\exp \{-(K x, x) / t\}$ as $\exp \{-(K x-K(0), x) / t\} \exp \{-(K(0), x) / t\}$ and use a subsequence of $P$ if necessary, it is easy to check that $\exp \{-(K Q x, Q x) / t\}$ converges to $\exp \{-(K x, x) / t\}$ in probability as $P \rightarrow I$ through $\mathscr{F} *$. From this fact and Step (ii) it follows that there exists a subsequence, still denoted by $P$ for convenience, such that

$$
\sigma_{P}(\cdot) \rightarrow(F \circ T)(\cdot) \exp \{-(K(\cdot),(\cdot)) / t\}
$$

in probability as $P \rightarrow I$ through $\mathscr{F} *$.

We choose a subsequence for which $\pi_{P} \rightarrow \pi$ a.e. as in Step (iii). For this subsequence we have $\sigma_{P} \rightarrow \sigma$ in probability, where $\sigma(x)=F \circ T(x) \exp \{-(K x, x) / t\}$; 
then there exists a subsubsequence for which $\sigma_{p} \rightarrow \sigma$ almost everywhere. Thus for this subsubsequence $\sigma_{P} \cdot \pi_{P} \rightarrow \sigma \cdot \pi$ a.e.

Now, let $\sigma_{2}(x)=\exp \left\{-|K x|^{2} / 2 t\right\}$, then $\sigma_{2} \circ Q \rightarrow \sigma_{2}$ in probability as $P \rightarrow I$ through $\mathscr{F}^{*}$. But

$$
J_{Q}(x)=\sigma_{P}(x) \times \exp \left\{-|P K Q x|^{2} / 2 t\right\} \pi_{P}(x), \quad x \in B .
$$

Hence combine the previous paragraph discussion there exists a subsubsequence, still denoted by $P$, such that

$$
J_{Q} \rightarrow J \text { almost everywhere }
$$

where $J(x)=F \circ T(x) \exp \left\{\left[-2(K x, x)-|K x|^{2}\right] / 2 t\right\}$ det $\left|I+K_{x}\right|$.

Step (v). Let us consider $J_{Q}$ in (1.6). Put $Q x=y$ and

$$
(I+P K P) y=z \text {. }
$$

Because the support of $F$ is contained in $b(K(0), R,\|\cdot\|)$, therefore to estimate $J_{Q}$, it suffices to consider those $y$ for which $\|z-K(0)\| \leqq R$. From (1.10), $y=z-P K P y$ $=z-P K y=(z-K(0))+(K(0)-K y)+(K y-P K y)$. But $\|z-K(0)\| \leqq R$. Also

$$
K y-K(0)=\int_{0}^{1} K_{t y} y d t
$$

therefore

$$
\|K y-K(0)\| \leqq \int_{0}^{1}\left\|K_{t y}\right\|_{B, B}\|y\| d t \leqq c\|y\| .
$$

Moreover $\|K y-P K y\|=\|(I-P) K y\| \leqq \beta|(I-P) K y|$; from the proof in Step (ii), there exists $P_{0}$ such that $P \geqq P_{0} \Rightarrow|(I-P) K y|<1 / \beta$ for all $y \in B$. Hence we have

$$
\|y\| \leqq\|z-K(0)\|+\|K(0)-K y\|+\|K y-P K y\| \leqq R+c\|y\|+1
$$

or $\|y\| \leqq(1+R) /(1-c)$. Therefore we have whenever $P \geqq P_{0}\|Q x\| \leqq(1+R) /(1-c)$ for all $x \in B$. It follows that whenever $P \geqq P_{0}$

$$
\left|J_{Q}(x)\right| \leqq b\|F\|_{\infty} \exp \left\{M t^{-1}(1+R) /(1-c)\right\} \exp \left\{\left|(Q K(0), x) t^{-1}\right|\right\} \text { for all } x \in B .
$$

Note that $\exp \left\{\left|(Q K(0), x) t^{-1}\right|\right\}$ is an integrable function because $Q K(0) \in H$. Moreover $\exp \left\{\left|(Q K(0), x) t^{-1}\right|\right\}$ converges in the mean to $\exp \left\{\left|(K(0), x) t^{-1}\right|\right\}$ as $P \rightarrow I$. Applying Lebesgue dominated convergence theorem, we have from (1.9)

$$
\lim _{P \rightarrow I \text { through } \mathscr{F}^{*}} \int_{B} J_{Q}(x) d p_{t}(x)=\int_{B} J(x) d p_{t}(x) .
$$

Finally from (1.8) and (1.11)

$$
\int_{B} F(y) d p_{t}(y)=\int_{B} F \circ T(x) g_{t}(x) d p_{t}(x) .
$$


The last assertion $p_{t}\left\{g_{t}>0\right\}=1$ follows from the fact that it is the product of two factors, one of which is the exponential function which is positive a.e., the other one is the determinant function which is bounded and bounded away from zero as shown in Step (ii).

The proof has now been completed. Q.E.D.

(c) The integral formula for transformations defined in a sphere. Let $\theta$ be a monotonic $C^{1}$-function from $[0, \infty]$ into $[0,1]$ such that

$$
\begin{aligned}
\theta(x)=1 & \text { when } 0 \leqq x \leqq 1, \\
& =0
\end{aligned}
$$

LeMMA I.6. Let $S=\left\{x \in B ;\left\|x-x_{0}\right\|<r\right\} \equiv b\left(x_{0}, r,\|\cdot\|\right)$ and let $K$ be a transformation from $S$ into $B$ of class $C^{1}$ with the following properties:

(0) $K\left(x_{0}\right)=0, K_{x_{0}}=0$.

(1) $K(S) \subset H$ and $K_{x}(B) \subset B^{*}, K_{x} \in \mathscr{B}_{1}(H, H)$ for all $x \in S$.

(2) The mapping $x \rightarrow K_{x}$ from $S$ (with the $B$ topology) into $\mathscr{B}\left(B, B^{*}\right)$ (with the uniform topology) is continuous, and from $S$ (with the $B$ topology) into $\mathscr{B}_{1}(H, H)$ (with the trace class norm topology).

(3) There exists a constant $M<\infty$ such that $\left\|K_{x}\right\|_{1} \leqq M$ for all $x \in S$.

Let $K_{\lambda}(x)=\theta\left(\lambda\left\|x-x_{0}\right\|^{2}\right) K(x), \lambda>(4 / r)^{2} . K_{\lambda}$ is globally defined if we let $K \equiv 0$ on $S^{c}$ (the complement of $S$ in $B$ ).

Then given any $\delta, 0<\delta<1$, there exists $\lambda_{0}>0$ such that whenever $\lambda>\lambda_{0}$, we have the following

(a) $K_{\lambda}$ satisfies all the properties of $K$ with $B$ replacing $S$,

(b) $\left\|\left(K_{\lambda}\right)_{x}\right\|_{H, H} \leqq \delta,\left\|\left(K_{\lambda}\right)_{x}\right\|_{B, B} \leqq \delta$ for all $x \in B$, and

(c) $\left\|K_{\lambda} x\right\|_{B^{*}} \leqq 1$ for all $x \in B$.

Proof. Straightforward verification. Q.E.D.

THEOREM I.7. Let $S=b\left(x_{0}, r,\|\cdot\|\right) \subset B$ and let $K$ be a transformation from $S$ into $B$ of class $C^{1}$ with the properties (0), (1), (2) and (3) of Lemma I.6. Let $T=I+K$. Then there exists $0<r_{0}<r$ such that $p_{t} \circ T$ and $p_{t}$, as measures on $b\left(x_{0}, r_{0},\|\cdot\|\right)$, are equivalent with

$$
\frac{d p_{t} \circ T}{d p_{t}}(x)=g_{t}(x), \quad x \in b\left(x_{0}, r_{0},\|\cdot\|\right) .
$$

Proof. Define $K_{\lambda}$ as in Lemma I.6 and choose $\lambda_{0}$ so that the conclusions of Lemma I.6 hold. Thus whenever $\lambda>\lambda_{0} K_{\lambda}$ satisfies the hypothesis of Theorem I.5. Put $r_{0}=\left(2 \lambda_{0}\right)^{-1 / 2}$ and apply Theorem I.5 to coinplete the proof. Q.E.D.

LEMMA I.8. Let $K$ be a continuous operator from $B$ into itself with image in $B^{*}$ such that $K \in \mathscr{B}_{1}(H, H)$ and $I+K$ is nonsingular from $B$ into itself. Let $h \in H$. Then for any integrable functional $F$ on $B$, we have

$$
\begin{aligned}
\int_{B} F(y) d p_{t}(y)= & \exp \left(-|h|^{2} / 2 t\right) \operatorname{det}|I+K| \int_{B} F(h+x+K x) \\
& \cdot \exp \left\{\left[-2(K x, x)-2(h, x)-|K x|^{2}-(K x, h)-(x, K h)\right] / 2 t\right\} d p_{t}(x) .
\end{aligned}
$$


Proof. Apply Theorem 3 of [15] by taking the representative of $n_{t}$ given in Corollary 1 of [8].

THEOREM I.9. The conclusion of Theorem I.7 remains valid if the hypotheses are the same except that $K\left(x_{0}\right)=0$ and $K_{x_{0}}=0$ are replaced by

$$
I+K_{x_{0}}: B \rightarrow B \text { is nonsingular. }
$$

Proof. Define $T_{2}: B \rightarrow B$ by $T_{2}=L_{a} \circ T_{x_{0}}$ where $L_{a}(y)=a+y$ and $a=K\left(x_{0}\right)$ $-K_{x_{0}}\left(x_{0}\right)$. Define $T_{1}=T_{2}^{-1} \circ T$, then $T=T_{2} \circ T_{1}$. It is easy to check that $T_{1}$ satisfies the hypotheses of Theorem I.7 and $T_{2}$ satisfies that of Lemma I.8. The conclusion then follows immediately. Q.E.D.

(d) Proof of Theorem I.4. By (ad-3) for each point $x_{0} \in U$ there exist a neighborhood $S\left(x_{0}\right)=b\left(x_{0}, r\left(x_{0}\right),\|\cdot\|\right) \subset U$ of $x_{0}$ and a constant $C\left(x_{0}\right)$ such that $\left\|T_{x}-I\right\|_{1}$ $\leqq C\left(x_{0}\right)$ for all $x \in S\left(x_{0}\right)$.

Let $K=T-I$. Then $K$ satisfies all the hypotheses of Theorem I.9 when it is restricted to each sphere $S\left(x_{0}\right)$. Therefore by Theorem I.9 for each point $x_{0} \in U$ there exists $S_{0}\left(x_{0}\right) \subset S\left(x_{0}\right)$ such that the transformation formula is true on any measurable subset of $S_{0}\left(x_{0}\right)$.

Clearly $U=\bigcup_{x_{0} \in U} S_{0}\left(x_{0}\right)$. But $B$ is separable, so there exist countable points $x_{1}, x_{2}, \ldots$ in $U$ such that $U=\bigcup_{n=1}^{\infty} S_{0}\left(x_{n}\right)$. Let $\Gamma_{1}=S_{0}\left(x_{1}\right), \Gamma_{n}=S_{0}\left(x_{n}\right)-\bigcup_{k=1}^{n-1} \Gamma_{k}$, $n \geqq 2$. Then $U=\bigcup_{k=1}^{\infty} \Gamma_{k}$ is a disjoint union of Wiener measurable subsets of $U$. On the other hand, $T$ is by assumption a homeomorphism from $U$ onto $V$, therefore $V=\bigcup_{k=1}^{\infty} T \Gamma_{k}$ is also a disjoint union of Wiener measurable subsets.

Now if $F$ is an integrable functional on $V$, then it is also integrable on each $T \Gamma_{k}$, hence

$$
\begin{aligned}
\int_{V} F(y) d p_{t}(y) & =\sum_{k=1}^{\infty} \int_{T \Gamma_{k}} F(y) d p_{t}(y)=\sum_{k=1}^{\infty} \int_{\Gamma_{k}} F \circ T(x) g_{t}(x) d p_{t}(x) \\
& =\int_{U} F \circ T(x) g_{t}(x) d p_{t}(x) .
\end{aligned}
$$

If $F$ is a Wiener measurable functional on $V$ such that $\int_{U} F \circ T(x) g_{t}(x) d p_{t}(x)$ exists, then each $\int_{\Gamma_{k}} F \circ T(x) g_{t}(x) d p_{t}(x)$, and hence each $\int_{T \Gamma_{k}} F(y) d p_{t}(y)$, and hence $\int_{V} F(y) d p_{t}(y)$ exists and the conclusion of the theorem follows from the previous paragraph. Q.E.D.

(e) Relations with other works. Our Theorem I.4 is a generalization of Theorem 4 of [9]. This is shown by the following

THEOREM I.10. If we have the assumption of Theorem 4 of [9], then there exists a Hilbert space $B$ with norm $\|\cdot\|$ such that $(i, H, B)$ is an abstract Wiener space and $K$ extends to $B$ such that $T=I+K$ is an admissible transformation from $B$ onto itself.

It is well known that $C$ ( $\equiv$ the Banach space consisting of the real-valued continuous functions on $[0,1]$ which vanish at 0 ) has no $\|\cdot\|_{\infty}$-equivalent norm which 
is $C^{1}$. Thus our Theorem I.4 can not be applied to the space $C$. However the following theorem is a partial generalization of the Theorem $\mathrm{V}$ of [3].

TheOREM I.11. Suppose $(i, H, B)$ is an abstract Wiener space and $B$ has a weaker norm $\|\cdot\|$ such that $\|x\|$ is of class $C^{1}$ off the origin in $B$. Let $B_{0}$ denote the normed linear space $(B,\|\cdot\| \mid \|)$. Suppose $U$ is a $\|\cdot\| \cdot \|$-open subset of $B$ and $T$ is a Fréchet differentiable transformation from $U$ (regarded as $\|\cdot\|$-open subset of $B$ ) into $B$ satisfying the following conditions:

(1) $T x-x \in H, T_{x}: B \rightarrow B$ nonsingular with $T_{x}-I \in \mathscr{B}\left(B_{0}, B^{*}\right)$ and $T_{x}-I \in \mathscr{B}_{1}(H, H)$ for all $x \in U$.

(2) The map $x \rightarrow T_{x}-I$ is continuous from $U$ (with the $\|\cdot\| \mid \|$-topology) into $\mathscr{B}\left(B_{0}, B^{*}\right)$ (with the uniform operator norm topology). It is also continuous from $U$ (with the $\||\cdot| \mid-$-topology) into $\mathscr{B}_{1}(H, H)$ (with the trace class norm topology).

Then

$$
\frac{d p_{t} \circ T}{d p_{t}}(x)=g_{t}(x), \quad x \in U
$$

II. Riemann-Wiener manifold. From now on we assume that the $B$-norm $\|\cdot\|$ has the following properties: (i) The function $\|\cdot\|$ is of class $C^{1}$ off the origin and (ii) $\|\cdot\|$ is $p_{1}$-square integrable over $B$. $H_{0}$ denotes the normed linear space $(H,\|\cdot\|)$.

(a) Definitions and properties. Let $\mathscr{W}$ be a $C^{k}$-differentiable manifold $(k \geqq 1)$ modelled on $B$. Suppose $(U, \varphi)$ and $(V, \psi)$ are two charts at $x \in \mathscr{W}$. Let $\left(R_{\varphi}\right)_{x}=$ $\varphi_{*, x}^{-1}(H),\left(R_{\psi}\right)_{x}=\psi_{*, x}^{-1}(H)$. If $\varphi \circ \psi^{-1}$ is admissible from $\psi(U \cap V)$ onto $\varphi(U \cap V)$, then $\left(R_{\varphi}\right)_{x}=\left(R_{\psi}\right)_{x}$; the equality here means they have the same point set and the same Hilbert structure.

Now suppose there exists an open covering $\left\{U_{\alpha}, \varphi_{\alpha}\right\}_{\alpha \in \Lambda}$ of $\mathscr{W}$ by charts such that, for any $\alpha, \beta \in \Lambda, \varphi_{\alpha} \circ \varphi_{\beta}^{-1}: \varphi_{\beta}\left(U_{\alpha} \cap U_{\beta}\right) \rightarrow \varphi_{\alpha}\left(U_{\alpha} \cap U_{\beta}\right)$ is admissible. A chart $(U, \varphi)$ in $\mathscr{W}$ is called compatible with respect to $\left\{\left(U_{\alpha}, \varphi_{\alpha}\right)\right\}_{\alpha \in \Lambda}$ if, for any $\alpha$, $\varphi_{\alpha} \circ \varphi^{-1}: \varphi\left(U \cap U_{\alpha}\right) \rightarrow \varphi_{\alpha}\left(U \cap U_{\alpha}\right)$ is admissible. For each point $x \in \mathscr{W}$ there is a Hilbertable vector space $R_{x}$ associated with $\left\{\left(U_{\alpha}, \varphi_{\alpha}\right)\right\}_{\alpha \in \Lambda}$. Precisely, let $x \in U_{\beta}$ for some $\beta \in \Lambda$, then we define $R_{x} \equiv\left(\varphi_{\beta}\right)_{*, x}^{-1}(H)$ and transport the Hilbert structure of $H$ to $R_{x}$ by $\left(\varphi_{\beta}\right)_{*, x}^{-1}$. By the above remark $R_{x}$ is completely determined by

$$
\left\{\left(U_{\alpha}, \varphi_{\alpha}\right)\right\}_{\alpha \in \Lambda} .
$$

Let $S L^{2}\left(R_{x} ; R\right)$ be the totality of continuous symmetric bilinear forms on $R_{x}$ and let $S L^{2}(R ; R)$ be the disjoint union of $S L^{2}\left(R_{x} ; R\right)$ over all $x \in \mathscr{W}$. Let c be the projection map from $S L^{2}(R ; R)$ into $\mathscr{W}$. Then by Proposition 2 , p. 36, of [12] there exists a unique $C^{j-1}$-structure on $S L^{2}(R ; R)$ such that $S L^{2}(R ; R)$ is a $C^{j-1}$ manifold modelled on $B \times S L^{2}(H ; R)$ and $\iota$ is a $C^{j-1}$ map from $S L^{2}(R ; R)$ into $\mathscr{W}$.

Definition II.1. A continuous bilinear form $\theta$ on $H$ is said to be nonsingular if there exists a continuous bilinear form $\eta$ on $H$ such that for some orthonormal basis $\left\{e_{k}\right\}_{k=1}^{\infty}$ of $H$, we have $\sum_{k=1}^{\infty} \theta\left[x, e_{k}\right] \eta\left[y, e_{k}\right]=\langle x, y\rangle$ for all $x, y \in H$. 
Notations. We use [, ] to denote element of $H \times H . \sigma$ denotes the bilinear form on $H$ given by $[h, k] \rightarrow\langle h, k\rangle$.

Definition II.2. A transformation $f$ from an open subset $U$ of $B$ into $L^{2}(H ; R)$ is said to be of type $\left(2, H_{0}\right)$ if it satisfies the following conditions:

(i) $f(x)$ is nonsingular and $f(x)-\sigma \in L^{2}\left(H_{0} ; R\right)$ for all $x \in U$.

(ii) $f$ is Fréchet differentiable at every point $x$ of $U . f^{\prime}(x) \in L^{3}\left(B, H_{0}, H_{0} ; R\right)$ for all $x \in U$ and the transformation $x \rightarrow f^{\prime}(x)$ from $U$ into $L^{3}\left(B, H_{0}, H_{0} ; R\right)$ is continuous.

(iii) $f^{\prime}$ is Fréchet differentiable at every point $x$ of $U . f^{\prime \prime}(x) \in L^{4}\left(B, B, H_{0}, H_{0} ; R\right)$ for all $x \in U$ and the transformation $x \rightarrow f^{\prime \prime}(x)$ from $U$ into $L^{4}\left(B, B, H_{0}, H_{0} ; R\right)$ is continuous.

REMARKS. (1) If $f$ is a transformation from $U$ into $L^{2}(H ; R)$ of type $\left(2, H_{0}\right)$, then $f^{\prime}(x)$ extends to $L^{3}(B ; \boldsymbol{R})$ and $f^{\prime \prime}(x)$ extends to $L^{4}(B ; R)$ for all $x \in U$. We denote the extensions by $f^{\prime}(x)^{\sim}$ and $f^{\prime \prime}(x)^{\sim}$, thus we have maps $f^{\prime \sim}$ from $U$ into $L^{3}(B ; \boldsymbol{R})$ and $f^{\prime \prime} \sim$ from $U$ into $L^{4}(B ; \boldsymbol{R})$ defined by $f^{\prime} \sim(x)=f^{\prime}(x)^{\sim}$ and $f^{\prime \prime} \sim(x)=f^{\prime \prime}(x) \sim$. $f^{\prime} \sim$ and $f^{\prime \prime} \sim$ are continuous by condition (ii) and (iii).

(2) Suppose $\bar{f}(x)$ is the bounded operator of $H$ corresponding to $f(x)$; then $\bar{f}(x)-I \in \mathscr{B}_{1}(H, H)$. This is a consequence of Proposition 0.1 and the condition (i). Similarly for $u, v \in B$,

$$
f^{\prime}(x)^{\sim}(u) \in \mathscr{B}_{1}(H, H) \text { and } f^{\prime \prime}(x)^{\sim}(u)(v) \in \mathscr{B}_{1}(H, H) .
$$

Definition II.3. Let $\mathscr{W}$ be a $C^{j}$-differentiable manifold $(j \geqq 1)$ modelled on $B$. Suppose there exists an open covering $\left\{\left(U_{\alpha}, \varphi_{\alpha}\right)\right\}_{\alpha \in \Lambda}$ of $\mathscr{W}$ by charts such that, for any $\alpha, \beta \in \Lambda, \varphi_{\alpha} \circ \varphi_{\beta}^{-1}: \varphi_{\beta}\left(U_{\alpha} \cap U_{\beta}\right) \rightarrow \varphi_{\alpha}\left(U_{\alpha} \cap U_{\beta}\right)$ is admissible. An assignment $\tau$ of a norm to each tangent space is continuous if for each compatible chart $(U, \varphi)$ with respect to $\left\{\left(U_{\alpha}, \varphi_{\alpha}\right)\right\}_{\alpha \in \Lambda}$ the function $x \rightarrow\left\|\varphi_{*, x}\right\|_{T_{x}(\mathscr{W}), B}$ is continuous on $U$, where $T_{x}(\mathscr{W})$ has the norm $\tau(x)$. By a Wiener-structure for $\mathscr{W}$ we mean a continuous assignment $\tau$ of a norm to each tangent space in such a way that for each point $x$ of $\mathscr{W}$ there exists a compatible chart $(U, \varphi)$ at $x$ such that

$$
c^{-1}\left\|\varphi_{*, y} u\right\| \leqq \tau(y)(u) \leqq c\left\|\varphi_{*, y} u\right\| \quad \text { for all } y \in U \text { and all } u \in T_{y}(\mathscr{W})
$$

where $c$ is a constant independent of $y$.

Notation. Suppose $S$ and $T$ are linear operators from $X$ into $Y . S \times T$ denotes the map $\left[x_{1}, x_{2}\right] \rightarrow\left[S x_{1}, T x_{2}\right]$ from $X \times X$ into $Y \times Y$.

Definition II.4. By a Riemann-Wiener manifold of class $C^{j}(j \geqq 3)$ we mean a triple $(\mathscr{W}, \tau, g)$ satisfying the following axioms.

RW-0. $\mathscr{W}$ is a $C^{j}$-differentiable manifold modelled on $B$.

RW-1. There exists an open covering $\left\{\left(U_{\alpha}, \varphi_{\alpha}\right)\right\}_{\alpha \in \Lambda}$ of $\mathscr{W}$ by charts such that, for any $\alpha$ and $\beta$ in $\Lambda, \varphi_{\beta} \circ \varphi_{\alpha}^{-1}: \varphi_{\alpha}\left(U_{\alpha} \cap U_{\beta}\right) \rightarrow \varphi_{\beta}\left(U_{\alpha} \cap U_{\beta}\right)$ is admissible.

RW-2. $\tau$ is a Wiener structure for $\mathscr{W} . g$ is a $C^{j-1}$-map from $\mathscr{W}$ into $S L^{2}(R ; R)$ such that $\lg (x)=x$ for all $x \in \mathscr{W}$ and, for each point $y$ in $\mathscr{W}$, (a) $g(y)$ is positive definite, (b) $g(y)$, as an inner product on $R_{y}$, determines its Hilbert space structure. 
RW-3. For each point $x_{0} \in \mathscr{W}$ there exists a compatible chart $(U, \varphi)$ at $x_{0}$ with respect to $\left\{\left(U_{\alpha}, \varphi_{\alpha}\right)\right\}_{\alpha \in \Lambda}$ such that the transformation

$$
x \rightarrow g\left(\varphi^{-1}(x)\right) \circ\left[\left(\varphi^{-1}\right)_{*, x} \times\left(\varphi^{-1}\right)_{*, x}\right]
$$

from $\varphi(U)$ into $L^{2}(H ; R)$ is of type $\left(2, H_{0}\right)$.

Notation. $\xi_{\varphi}(y) \equiv g(y) \circ\left[\varphi_{*, y}^{-1} \times \varphi_{*, y}^{-1}\right]$. Then the transformation in RW-3 is $\xi_{\varphi} \circ \varphi^{-1}$.

Proposition II.5. (a) For each $x$ in $\mathscr{W},\left(R_{x}, T_{x}(\mathscr{W})\right)$ is an abstract Wiener space with inner products $\langle,\rangle_{x}=g(x)$ and norm $\tau(x)$ for $R_{x}$ and $T_{x}(\mathscr{W})$ respectively.

(b) If $n(x)_{t}$ is the canonical normal distribution on $R_{x}$, then $n(x)_{t}$ is equivalent to $n_{t} \circ\left(\varphi_{*, x}^{-1}\right)^{*}$, where $\varphi$ is given by $\mathrm{RW}-3$.

Proof. (a) Suppose $x \in U$, and $(U, \varphi)$ is a compatible chart at $x$. Define $\langle u, v\rangle_{\varphi}$ $=\left\langle\varphi_{*, x} u, \varphi_{*, x} v\right\rangle$ for $u, v \in R_{x}$ and $\|y\|_{\varphi}=\left\|\varphi_{*, x} y\right\|$ for $y \in T_{x}(\mathscr{W})$.

Then $\left(R_{x}, T_{x}(\mathscr{W})\right)$ is an abstract Wiener space under the inner product $\langle,\rangle_{\varphi}$ and norm $\|\cdot\|_{\varphi}$ for $R_{x}$ and $T_{x}(\mathscr{W})$ respectively. But by $\mathrm{RW}-2\langle,\rangle_{\varphi}$ is equivalent to $\langle,\rangle_{x}$ and $\|\cdot\|_{\varphi}$ is equivalent to $\tau(x)$. It is easy to see that $\tau(x)$ is a measurable norm over $\left(R_{x},\langle,\rangle_{x}\right)$. Therefore $\left(R_{x}, T_{x}(\mathscr{W})\right)$ is an abstract Wiener space with structure $g(x)$ and $\tau(x)$ respectively.

(b) Apply Theorem 3 of [15]. Q.E.D.

(b) Examples.

EXAmple 1. Any open set in $B$, with $\tau$ given by the norm of $B$ and $g$ by the inner product of $H$, is a Riemann-Wiener manifold.

ExAmple 2. Let $(i, \boldsymbol{H}, \boldsymbol{B})$ be an abstract Wiener space. Assume that $\|\cdot\|$ is a function of class $C^{j}(j \geqq 3)$ off the origin. Let $\mathscr{W}=\{x \in \boldsymbol{B} ;\|x\|=1\}$ be the unit sphere of $\boldsymbol{B}$. Let $\boldsymbol{\tau}$ and $g$ be induced by the $\boldsymbol{B}$-norm and $\boldsymbol{H}$-inner product respectively. Then $(\mathscr{W}, \tau, g)$ is a Riemann-Wiener manifold.

(c) Riemannian manifolds associated with Riemann-Wiener manifold.

THEOREM II.6. For each point $x \in \mathscr{W}$, there exists a subset $R(x)$ of $\mathscr{W}$ containing $x$ such that

(1) $R(x)$ is a separable topological space and $R(x) \hookrightarrow \mathscr{W}$ is continuous.

(2) $R(x)$ is a $C^{\infty}$-differentiable manifold modelled on $H$.

(3) $T_{y}(R(x))=R_{y}$ for all $y \in R(x)$, and

(4) $g$ is a Riemannian metric on $R(x)$, hence $(R(x), g)$ is a Riemannian manifold.

Proof. Let $(U, \varphi)$ be a compatible chart at $x$, define $R(x)=\varphi^{-1}((\varphi(x)+H) \cap \varphi(U))$ and let $R(x)$ receive the topology from $(\varphi(x)+H) \cap \varphi(U)$ with $H$-topology by the map $\varphi$. Note that $(\varphi(x)+H) \cap \varphi(Y)=(\varphi(U)-\varphi(x)) \cap H+\varphi(x)$, therefore $(\varphi(x)$ $+H) \cap \varphi(U)$ is an open set in $H$. Define a global chart $\psi$ from $R(x)$ into $H$ by $\psi(y)=\varphi(y)-\varphi(x)$. Now the conclusion of this theorem becomes obvious. Q.E.D.

REMARKS. (i) The set $R(x)$ constructed in the proof of the above theorem depends on the chart $(U, \varphi)$. However, if we use another chart $\left(U_{1}, \varphi_{1}\right)$ then we 
have the following relation. Let $R_{1}(x)$ be the corresponding set to $\left(U_{1}, \varphi_{1}\right)$, then $R_{1}(x) \cap U=R(x) \cap U_{1}$. In other words, $R_{1}(x) \cap\left(U \cap U_{1}\right)=R(x) \cap\left(U \cap U_{1}\right)$. This means that in the intersection of $U$ and $U_{1}, R(x)$ and $R_{1}(x)$ are the same point set.

(ii) We note that $R(x)$ is dense in $U$ with the $\mathscr{W}$-topology if they correspond to each other in the proof of the theorem.

Examples. (i) Let $U$ be an open subset of $B$. $U$ is a Riemann-Wiener manifold. For each point $x \in U, R(x)=(x+H) \cap U$. Thus $R(x)$ is the intersection of $U$ with a coset of $H$ in $B$. If $U=B$, then $R(x)$ is exactly the coset of $H$ in $B$ containing $x$.

(ii) Let $\mathscr{W}$ be the unit sphere of $\boldsymbol{B}$ (Example 2 of $\S(\mathrm{b}))$. Let $(U, \varphi)$ be a chart at $x$. If we take the chart $(U, \varphi)$ for the construction of $R(x)$, then

$$
R(x)=\{x+h \in \mathscr{W} ; h \in H\} \cap U .
$$

(d) Construction of a spray on $\mathscr{W}$ associated with $g$. Let $x_{0}$ be a fixed point of $\mathscr{W}$ and $(U, \varphi)$ a fixed chart at $x_{0}$ given by RW-3. From Theorem II.6, we know that there exists a Riemannian manifold $\left(R\left(x_{0}\right), g\right)$ containing $x_{0}$ modelled on the Hilbert space $H$. Let $\nabla$ be the Levi-Civita differential of $\left(R\left(x_{0}\right), g\right)$ given by the Fundamental Theorem of Riemannian Geometry. In the following discussion we will drop the index $\varphi$ in $\xi_{\varphi}$; we denote by $\xi$ the linear operator of $H$ corresponding to $\xi$.

Let $\left\{e_{j}\right\}_{j=1}^{\infty}$ be a fixed orthonormal basis of $H$. Let $\tilde{e}_{j}$ denote the vector field on $\left(R\left(x_{0}\right), g\right)$ defined by $\tilde{e}_{j}(x)=e_{j}(x) \equiv \varphi_{*, x}^{-1}\left(e_{j}\right), x \in R\left(x_{0}\right)$. For each point $x \in R\left(x_{0}\right)$ we define a bilinear map $\Gamma(x)$ from $R_{x} \times R_{x}$ into $R_{x}$ by $\Gamma(x)\left(e_{i}(x), e_{j}(x)\right)=\left(\nabla_{\tilde{e}_{i}} \tilde{e}_{j}\right)(x)$.

A simple calculation shows that for all $u, v, w \in H$ we have

$$
\begin{aligned}
2 \xi(x)\left(u, \varphi_{*, x} \Gamma(x)\left(\varphi_{*, x}^{-1}(w), \varphi_{*, x}^{-1}(v)\right)\right)=\left\langle\left(\xi \circ \varphi^{-1}\right)^{\prime}(\varphi(x))(w) v, u\right\rangle \\
+\left\langle\left(\xi \circ \varphi^{-1}\right)^{\prime}(\varphi(x))(v) u, w\right\rangle-\left\langle\left(\xi \circ \varphi^{-1}\right)^{\prime}(\varphi(x))(u) w, v\right\rangle .
\end{aligned}
$$

(2.1) defines uniquely a bilinear transformation $\Gamma(x): R_{x} \times R_{x} \rightarrow R_{x}$.

A curve $r$ on $\left(R\left(x_{0}\right), g\right)$ is called a geodesic if $\nabla_{r^{\prime}(t)} r^{\prime}(t)=0$. Using (2.1) it is easily checked that $r$ is a geodesic iff

$$
r^{\prime \prime}(t)+\Gamma(r(t))\left(r^{\prime}(t), r^{\prime}(t)\right)=0 .
$$

We will refer to (2.2) as the geodesic differential equation for the Riemannian manifold $\left(R\left(x_{0}\right), g\right)$.

Therefore for each point $x$ in $R\left(x_{0}\right)$, we have the exponential map at $x$, exp $x$, which is defined in the well-known way. In general $\exp x$ is defined only in some open neighborhood of the origin in $R_{x}$ (in the $g(x)$-topology); and to extend exp $x$ to a continuous function from some open neighborhood of the origin in $T_{x}(\mathscr{W})$ (in the $\tau(x)$-topology) into some open neighborhood of $x$ is by no means easy. Even in case $R\left(x_{0}\right)$ is complete so that $\exp x$ is defined on the whole space $R_{x}$, the simplest way to extend $\exp x$ continuously into $T_{x}(\mathscr{W})$ is to show that it continuous in $R_{x}$ with respect to the $\tau(x)$-topology. However, this is not obvious. 
Because of the difficulties of the last paragraph, we will use another approach. Namely, we will construct a spray $\chi$ on $\mathscr{W}$ by means of $g$. Then we use this spray to define the exponential map. This exponential map will turn out to be of class $C^{k}(k \geqq 1)$.

We will work locally. Thus let $(U, \varphi)$ be a chart given by RW-3. Moreover we will slur over the distance between $x \in U$ and $\varphi(x) \in B$; therefore we assume that $U$ is an open subset of $B$. The tangent bundle of $U$ is $U \times B$ and the double tangent bundle is $(U \times B) \times(B \times B)$. A spray on $U$ is given by the principal part $\chi: U \times B$ $\rightarrow B \times B$ satisfying $\chi(x, v)=\left(v,-\chi_{0}(x, v)\right)$ and $\chi_{0}(x, s v)=s^{2} \chi_{0}(x, v), s \in R$. We will construct $\chi_{0}: U \times B \rightarrow B$.

For fixed $v, w \in B$ consider the function

$$
\xi^{\prime}(x)^{\sim}(v, w, \cdot)+\xi^{\prime}(x)^{\sim}(w, \cdot, v)-\xi^{\prime}(x)^{\sim}(\cdot, v, w)
$$

on $B$. By (ii) of Definition II.2 this function is a continuous linear transformation from $B$ into $R$. Therefore it defines an element $\Phi(x ; v, w)$ in $B^{*}$, namely,

$$
\Phi(x ; v, w)=\xi^{\prime}(x)^{\sim}(v, w, \cdot)+\xi^{\prime}(x)^{\sim}(w, \cdot, v)-\xi^{\prime}(x)^{\sim}(\cdot, v, w) .
$$

Define a map $\Phi(x): B \times B \rightarrow B^{*}$ by

$$
\Phi(x)(v, w)=\Phi(x ; v, w) .
$$

It follows that from (2.3) and (2.4) we have

Proposition II.7. $\Phi(x)$ is a continuous bilinear transformation. In fact

$$
\|\Phi(x)\|_{B, B ; B^{*}} \leqq 3\left\|\xi^{\prime}(x)^{\sim}\right\|_{B^{3} ; R} .
$$

Now we define a map $\tilde{\Gamma}(x): B \times B \rightarrow B$ by

$$
\tilde{\Gamma}(x)(u, v)=2^{-1} \bar{\xi}(x)^{-1} \Phi(x ; u, v) .
$$

Proposition II.8. $\tilde{\Gamma}(x)(B \times B) \subset B^{*}$. Moreover $\tilde{\Gamma}(x)(u, v)=\Gamma(x)(u, v)$ if $u, v \in H$, i.e. $\tilde{\Gamma}(x)$ extends $\Gamma(x)$.

Proof. We know that, for all $u, v \in B, \Phi(x ; u, v) \in B^{*}$. By (i) of Definition II.2 $(\xi(x)-I)(H) \subset B^{*}$ and $\xi(x)$ is nonsingular, therefore $\xi(x)\left(B^{*}\right)=B^{*}$. It follows that $\xi(x)^{-1}\left(B^{*}\right)=B^{*}$, whence $\tilde{\Gamma}(x)(B \times B) \subset B^{*}$. The second assertion is obvious. Q.E.D.

Finally we define a map $\chi_{0}: U \times B \rightarrow B$ by

$$
\chi_{0}(x, v)=\tilde{\Gamma}(x)(v, v)
$$

and let $\chi: U \times B \rightarrow B \times B$ be defined by

$$
\chi(x, v)=\left(v,-\chi_{0}(x, v)\right) .
$$

Proposition II.9. (1) $\chi$ is a spray.

$$
\begin{aligned}
\chi_{0}^{\prime}(x, v)(u, w)= & -2^{-1} \xi(x)^{-1}\left\{\xi^{\prime}(x)\left(u, \xi(x)^{-1} \Phi(x ; v, v), \cdot\right)\right\} \\
& +2^{-1} \xi(x)^{-1}\left(D_{x} \Phi(x ; v, v)(u)\right)+\xi(x)^{-1} \Phi(x ; v, w),
\end{aligned}
$$

where $D_{x} \Phi$ denotes the derivative with respect to the variable $x$. 
(3) $\chi$ is of class $C^{j-2}(j \geqq 3)$.

Proof. (1) is clear since

$$
\begin{aligned}
\chi_{0}(x, s v) & =\tilde{\Gamma}(x)(s v, s v)=2^{-1} \xi(x)^{-1} \Phi(x ; s v, s v) \\
& =2^{-1} \xi(x)^{-1}\left(s^{2} \Phi(x ; v, v)\right)=s^{2} 2^{-1} \xi(x)^{-1} \Phi(x ; v, v)=s^{2} \chi_{0}(x, v)
\end{aligned}
$$

(2) follows by calculation.

(3) follows from (2) and the fact that $\xi$ is of class $C^{j-1}(j \geqq 3)$. Q.E.D.

(e) Study of the exponential map. As constructed in the last section, we have a spray on $\mathscr{W}$ which is locally given by $\chi(x, v)=\left(v,-\chi_{0}(x, v)\right)$, where $\chi_{0}(x, v)=$ $\tilde{\Gamma}(x)(v, v)$. Let $\beta(t)=(r(t), \theta(t))$ be the unique integral curve on $\mathscr{W}$ of $\chi$ with initial condition $\beta(0)=\left(x_{0}, v_{0}\right), x_{0} \in \mathscr{W}, v_{0} \in T_{x_{0}}(\mathscr{W})$. If $v_{0} \in R_{x_{0}}$, then it can (and will later) be shown that $r(t) \in R\left(x_{0}\right)$ and $\theta(t) \in R_{r(t)}$ for all $t$ in the domain of $\beta$. Since $\beta$ is an integral curve of $\chi$, it must satisfy $\beta^{\prime}(t)=\chi(\beta(t))$. Therefore $r^{\prime}(t)=\theta(t), \theta^{\prime}(t)=$ $-\tilde{\Gamma}(r(t))(\theta(t), \theta(t))=-\Gamma(r(t))(\theta(t), \theta(t))$. It follows immediately that

$$
r^{\prime \prime}(t)+\Gamma(r(t))\left(r^{\prime}(t), r^{\prime}(t)\right)=0 .
$$

This is the geodesic differential equation for $\left(R\left(x_{0}\right), g\right)$ in (2.2).

Let Exp denote the exponential map associated with $\chi$ and $\exp x$ the restriction of Exp to the tangent space $T_{x}(\mathscr{W})$. The following lemma is well known.

Lemma II.10. For each point $x \in \mathscr{W}$ there exist open sets $V(x)$ and $U(x) ; V(x)$ is a star-like open subset of $T_{x}(\mathscr{W})$ with respect to the origin and $U(x)$ is an open neighborhood of $x$ in $\mathscr{W}$, such that $\exp x$ is a $C^{j-2}$ homeomorphism from $V(x)$ onto $U(x)$, $j \geqq 3$.

Fact II.11. By taking a smaller neighborhood of $x$ if necessary, we may assume the following two properties:

$(\exp x)_{v}$, the derivative of $\exp x$ at $v$, is nonsingular from $T_{x}(\mathscr{W})$ into $T_{\exp x(v)}(\mathscr{W})$ for all $v \in V(x)$.

For each point $x$ in $\mathscr{W}, U(x) \subset U$, where $U$ is the domain of a chart $(U, \varphi)$ at $x$ given by $\mathrm{RW}-3$.

PROPOSITION II.12. $\exp x\left(V(x) \cap R_{x}\right)=U(x) \cap R(x)$ for all $x \in \mathscr{W}$.

Proof. By (2.9) we may assume $U(x)$ to be an open set in $B$. Then the assertion is equivalent to $\exp x(V(x) \cap H)=(x+H) \cap U(x)$. By the Taylor formula $r(t)=$ $r(0)+t r^{\prime}(0)+t^{2} \int_{0}^{1}(1-s) r^{\prime \prime}(t s) d s$. Hence if $r(0)=x, r^{\prime}(0)=v$, then

$$
\begin{aligned}
\exp x(v)=r(1) & =x+v+\int_{0}^{1}(1-s) r^{\prime \prime}(s) d s \\
& =x+v+\int_{0}^{1}(1-s) \tilde{\Gamma}(r(s))\left(r^{\prime}(s), r^{\prime}(s)\right) d s .
\end{aligned}
$$


Proposition II.8 and condition (iii) of the Definition II.2 imply that

$$
\int_{0}^{1}(1-s) \tilde{\Gamma}(r(s))\left(r^{\prime}(s), r^{\prime}(s)\right) d s \in B^{*}
$$

This yields the desired conclusion easily. Q.E.D.

Suppose $U\left(x_{0}\right)$ and $U\left(y_{0}\right)$ have nonempty intersection and are both contained in a chart $(U, \varphi)$ such that $\xi_{\varphi} \circ \varphi^{-1}$ is of type $\left(2, H_{0}\right)$ on $\varphi(U)$. We may assume $U$ to be an open set in $B$, therefore $U\left(x_{0}\right)$ and $U\left(y_{0}\right)$ are open sets in $B$ with nonempty intersection. $V\left(x_{0}\right)$ and $V\left(y_{0}\right)$ are by construction star-like open sets in $B$ containing the origin.

Notations. $U\left(x_{0}, y_{0}\right)=U\left(x_{0}\right) \cap U\left(y_{0}\right), W\left(x_{0}\right)=\exp x_{0}^{-1}\left(U\left(x_{0}, y_{0}\right)\right)$ and

$$
W\left(y_{0}\right)=\exp y_{0}^{-1}\left(U\left(x_{0}, y_{0}\right)\right) \text {. }
$$

THEOREM II.13. If $y_{0}-x_{0} \in H$ then $\exp x_{0}^{-1} \circ \exp y_{0}$ is an admissible transformation from $W\left(y_{0}\right)$ onto $W\left(x_{0}\right)$.

Proof. Let $T=\exp x_{0}^{-1} \circ \exp y_{0}$ then $T$ is clearly a homeomorphism of class $C^{j-2}(j-2 \geqq 1)$ from $W\left(y_{0}\right)$ onto $W\left(x_{0}\right)$. We prove this theorem by a series of claims.

(i) $T u-u \in H$ for all $u \in W\left(y_{0}\right)$.

From (2.10)

$$
\exp y_{0}(u)=y_{0}+u+\int_{0}^{1}(1-s) \tilde{\Gamma}(r(s))\left(r^{\prime}(s), r^{\prime}(s)\right) d s
$$

where $r^{\prime \prime}(t)+\tilde{\Gamma}(r(t))\left(r^{\prime}(t), r^{\prime}(t)\right)=0, r(0)=y_{0}$ and $r^{\prime}(0)=u$. Therefore

$$
\exp y_{0}(u)-u-y_{0} \in B^{*} \text {. }
$$

On the other hand, there exists $w \in W\left(x_{0}\right)$ such that $\exp x_{0}(w)=\exp y_{0}(u)$, hence $\exp x_{0}^{-1}\left(\exp y_{0}(u)\right)-\exp y_{0}(u)=w-\exp x_{0}(w)=-\left(\exp x_{0}(w)-w\right)$.

By the same reason as (2.11) we have $\exp x_{0}(w)-w-x_{0} \in B^{*}$, it follows that

$$
\exp x_{0}^{-1}\left(\exp y_{0}(u)\right)-\exp y_{0}(u)+x_{0} \in B^{*} \text {. }
$$

Now

$$
\begin{aligned}
T u-u & =\exp x_{0}^{-1}\left(\exp y_{0}(u)\right)-u \\
& =\left\{\exp x_{0}^{-1}\left(\exp y_{0}(u)\right)-\exp y_{0}(u)+x\right\}+\left\{\exp y_{0}(u)-u-y_{0}\right\}+\left\{y_{0}-x_{0}\right\} .
\end{aligned}
$$

By (2.11) and (2.12) the first and the second term are in $B^{*}$. By the assumption $y_{0}-x_{0} \in H$, therefore $T u-u \in H$ for all $u \in W\left(y_{0}\right)$.

(ii) $T_{u}$ is nonsingular from $B$ onto itself for all $u \in W\left(y_{0}\right)$.

This follows from (2.8).

(iii) $\left(\exp y_{0}\right)_{u}-I \in \mathscr{B}\left(B, B^{*}\right)$ for all $u \in W\left(y_{0}\right)$. 
We use $r(s, u)$ to denote the integral curve of $\chi$ with initial condition $r(0, u)=y_{0}$, $r^{\prime}(0, u)=u$. We know that $r$ is differentiable from $J_{0} \times V\left(y_{0}\right)$ into $U\left(y_{0}\right)$, where $J_{0}$ is some open interval containing $[0,1]$. From $(2.10)$

$$
\exp y_{0}(u)=y_{0}+u+\int_{0}^{1}(1-s) \tilde{\Gamma}(r(s, u))\left(r^{\prime}(s, u), r^{\prime}(s, u)\right) d s .
$$

Putting $\tilde{\Gamma}(x)(u, v)=2^{-1} \xi(x)^{-1} \Phi(x ; u, v)$ into the above expression, we obtain that

$$
\exp y_{0}(u)=y_{0}+u+2^{-1} \int_{0}^{1}(1-s) \xi(r(s, u))^{-1} \Phi\left(r(s, u) ; r^{\prime}(s, u), r^{\prime}(s, u)\right) d s
$$

Since $\xi$ is of type $\left(2, H_{0}\right)$ it follows that $\left(\left(\exp y_{0}\right)_{u}-I\right)(B) \subset B^{*}$ by a direct computation and using Proposition II.9. By Proposition $0.2\left(\exp y_{0}\right)_{u}-I \in \mathscr{B}\left(B, B^{*}\right)$.

(iv) $T_{u}-I \in \mathscr{B}\left(B, B^{*}\right)$ for all $u \in W\left(y_{0}\right)$.

$T_{u}=\left(\exp x_{0}\right)_{T u}^{-1}\left(\exp y_{0}\right)_{u}, u \in W\left(y_{0}\right)$. Hence

$$
\begin{aligned}
T_{u}-I & =\left(\exp x_{0}\right)_{T u}^{-1}\left(\exp y_{0}\right)_{u}-I \\
& =\left(\exp x_{0}\right)_{T u}^{-1}\left\{\left(\exp y_{0}\right)_{u}-\left(\exp x_{0}\right)_{T u}\right\} \\
& =\left(\exp x_{0}\right)_{T u}^{-1}\left\{\left[\left(\exp y_{0}\right)_{u}-I\right]-\left[\left(\exp x_{0}\right)_{T u}-I\right]\right\} .
\end{aligned}
$$

It follows from the previous step that $T_{u}-I \in \mathscr{B}\left(B, B^{*}\right)$.

(v) The mapping $u \rightarrow T_{u}-I$ is continuous from $W\left(y_{0}\right)$ (with the B-topology) into $\mathscr{B}\left(B, B^{*}\right)$ (with the uniform topology).

By (iii) $\left(\exp x_{0}\right)_{T u}\left(B^{*}\right)=B^{*}$. Therefore by $(2.14)$ it is sufficient to show that the mapping $u \rightarrow\left(\exp y_{0}\right)_{u}-I$ is continuous from $W\left(y_{0}\right)$ into $\mathscr{B}\left(B, B^{*}\right)$.

However this is implied by RW-3. We have shown that $T$ satisfies the assumption of Proposition I.3; therefore by its conclusion $T$ is admissible. Q.E.D.

In the general case by $(2.9)$ there exist charts $(U, \varphi)$ and $(V, \psi)$ at $x_{0}$ and $y_{0}$ respectively such that $U \supset U\left(x_{0}\right)$ and $V \supset U\left(y_{0}\right)$.

Notations. $S\left(x_{0}\right)=\varphi_{*, x_{0}}\left(W\left(x_{0}\right)\right), S\left(y_{0}\right)=\psi_{*, y_{0}}\left(W\left(y_{0}\right)\right)$.

THEOREM II.14. If $\psi\left(y_{0}\right)-\varphi\left(x_{0}\right) \in H$, then $\varphi_{*, x_{0}} \circ \exp x_{0}^{-1} \circ \exp y_{0} \circ \psi_{*, y_{0}}^{-1}$ is admissible from $S\left(y_{0}\right)$ onto $S\left(x_{0}\right)$.

Proof. Since $\varphi$ and $\psi$ are compatible, $\varphi \circ \psi^{-1}$ is admissible. The proof of this theorem is the same as that of Theorem II.13 with simple modifications and changes. We omit the details. Q.E.D.

III. Study of locally defined measures $q_{t}(x, \cdot)$ on $\mathscr{W}$.

(a) Statement of the second main result Theorem III.2.

Definition III.1. (1) For each point $x \in \mathscr{W}$ and $t>0$ we define a measure $q_{t}(x, \cdot)$ on the Borel field of $U(x)$ by

$$
q_{t}(x, E)=p_{t}^{(x)}\left(0, \exp x^{-1}(E)\right)
$$

where $E$ is a Borel subset of $U(x), p_{t}^{(x)}$ is the Wiener measure on $T_{x}(\mathscr{W})$ corresponding to the canonical normal distribution on $R_{x}$ with parameter $t$. 
(2) For each point $x \in \mathscr{W}$ and $t>0$, and each chart $(U, \varphi)$ at $x$ given by RW-3 such that $U(x) \subset U$ we define

$$
r_{t}^{(\varphi)}(x, E)=p_{t}\left(0, \varphi_{*, x} \exp x^{-1}(E)\right)
$$

where $E$ is a Borel subset of $U(x)$.

THEOREM III.2. Let $x$ and $y$ be two points in $\mathscr{W}$ such that $U(x)$ and $U(y)$ have nonempty intersection. Then $q_{t}(x, \cdot)$ and $q_{s}(y, \cdot)$, as measures on $U(x) \cap U(y)$, are equivalent if and only if $t=s$ and $d_{g}(x, y)<\infty$. Otherwise they are mutually singular. Here $d_{g}$ (defined in the next section) is the almost-metric on $\mathscr{W}$ determined by $g$.

Let $\varphi$ and $\psi$ be two charts at $x$ and $y$ respectively as in the definition of $r_{t}^{(\varphi)}(x, \cdot)$ and $r_{s}^{(\psi)}(y, \cdot)$.

Lemma III.3. Suppose $U(x) \cap U(y)$ is nonempty. Then as measures on $U(x) \cap U(y)$, $q_{t}(x, \cdot)$ and $q_{s}(y, \cdot)$ are equivalent if and only if $r_{t}^{(\varphi)}(x, \cdot)$ and $r_{s}^{(\psi)}(y, \cdot)$ are equivalent. The assertion is still true if "equivalent" is replaced by " mutually singular".

Proof. By Proposition II.5(b), $p_{t}^{(x)}$ is equivalent to $p_{t} \circ \varphi_{*, x}$ as measures on $T_{x}(\mathscr{W})$. Therefore they are also equivalent as measures on $V(x)$, and hence as measures on $\exp x^{-1}(U(x) \cap U(y))$. It follows that

$$
p_{t}^{(x)} \circ \exp x^{-1} \text { and } p_{t} \circ \varphi_{*, x} \circ \exp x^{-1}
$$

are equivalent as measures on $U(x) \cap U(y)$. But $q_{t}(x, \cdot)=p_{t}^{(x)} \circ \exp x^{-1}$ and $r_{t}^{(\varphi)}(x, \cdot)=p_{t} \circ \varphi_{*, x} \circ \exp x^{-1}$, hence $q_{t}(x, \cdot)$ and $r_{t}^{(\varphi)}(x, \cdot)$ are equivalent as measures on $U(x) \cap U(y)$. Similarly $q_{s}(y, \cdot)$ and $r_{s}^{(\psi)}(y, \cdot)$ are equivalent as measures on $U(x) \cap U(y)$. Now this lemma becomes obvious. Q.E.D.

THEOREM III.4. Suppose $x$ and $y$ are two points in $\mathscr{W}$ such that $U(x)$ and $U(y)$ have nonempty intersection. Then $r_{t}^{(\omega)}(x, \cdot)$ and $r_{s}^{(\psi)}(y, \cdot)$, as measures on $U(x) \cap U(y)$ are equivalent if and only if $t=s$ and $\psi(y)-\varphi(x) \in H$; otherwise they are mutually singular.

Proof. Case 1. $t=s$ and $\psi(y)-\varphi(x) \in H \Rightarrow r_{t}^{(\varphi)}(x, \cdot)$ is equivalent to $r_{s}^{(\psi)}(y, \cdot)$. This follows from Theorem II.14 and Theorem I.4.

Case 2. $t \neq s$ and $\psi(y)-\varphi(x) \in H \Rightarrow r_{t}^{(\varphi)}(x, \cdot)$ and $r_{s}^{(\psi)}(y, \cdot)$ are mutually singular. By Case $1, r_{t}^{(\omega)}(x, \cdot)$ is equivalent to $r_{t}^{(\psi)}(y, \cdot)$. On the other hand $p_{t}$ and $p_{s}$ are mutually singular by Proposition 1 of [10], hence $p_{t} \circ \psi_{*, y} \circ \exp y^{-1}$ and

$$
p_{s} \circ \psi_{*, y} \circ \exp y^{-1}
$$

are mutually singular. Therefore $r_{t}^{(\psi)}(y, \cdot)$ and $r_{s}^{(\psi)}(y, \cdot)$ are mutually singular, so $r_{t}^{(\varphi)}(x, \cdot)$ and $r_{s}^{(\Downarrow)}(y, \cdot)$ are mutually singular.

Case 3. $\psi(y)-\varphi(x) \in B \cap H^{c} \Rightarrow r_{t}^{(\varphi)}(x, \cdot)$ and $r_{s}^{(\psi)}(y, \cdot)$ are mutually singular for any $t, s>0$. 
Let $T=\varphi_{*, x} \circ \exp x^{-1} \circ \exp y \circ \psi_{*, y}^{-1}$. Let $L_{a}$ denote the translation by $a=\varphi(y)$ $-\psi(x)$. Define $J=L_{-a} \circ T$. Using the same argument as in Theorem II.13 it can be checked easily that $J$ is admissible from $S(y)$ onto $L_{-a}(S(x))$, where $S(z)$ are notations used in $\S I I(e) z=x, y$. Finally we apply Proposition 1 of [10] and our Theorem I.4 to finish the proof. Q.E.D.

THEOREM III.5. Suppose $x$ and $y$ are two points in $\mathscr{W}$ such that $U(x)$ and $U(y)$ have nonempty intersection. Then $q_{t}(x, \cdot)$ and $q_{s}(y, \cdot)$, as measures on $U(x) \cap U(y)$, are equivalent if and only if $t=s$ and $\psi(y)-\varphi(x)$ is in $H$ for some charts $\varphi$ and $\psi$ at $x, y$ respectively, where $r_{t}^{(\varphi)}$ and $r_{s}^{(\psi)}$ can be defined.

Proof. Easy consequence of Lemma III.3 and Theorem III.4. Q.E.D.

(b) The almost-metric $d_{g}$ on $\mathscr{W}$. Assume that $\mathscr{W}$ is connected. Take the component of $x$ if necessary, we may assume that the chart $(U, \varphi)$ in RW-3 is connected.

Definition III.6. (1) Let $r$ be a piecewise differentiable curve from $[a, b] \subset \boldsymbol{R}$ into $\mathscr{W}$. We define arclength $L_{g}(r)$ of $r$ with respect to $g$ by

$$
L_{g}(r)=\int_{a}^{b}\left\{g(r(t))\left(r^{\prime}(t), r^{\prime}(t)\right)\right\}^{1 / 2} d t .
$$

Here we use the convention $g(x)(u, u)=\infty$ if $u \in T_{x}(\mathscr{W}) \cap R_{x}^{c}$.

(2) Define $d_{g}: \mathscr{W} \times \mathscr{W} \rightarrow[0, \infty]$ by $d_{g}(x, y)=\inf L_{g}(r)$, where inf runs over the (nonempty) family of all piecewise differentiable curves joining $x$ and $y$.

REMARK. For any two points $x$ and $y$ in $\mathscr{W}, d_{g}(x, y)$ may be $\infty$. In the later part of this section we will study $d_{g}$ in more detail and give a necessary and sufficient condition for $d_{g}(x, y)<\infty$.

EXAMPLE. Let $U$ be a convex open subset of $B$ with $\tau(x)=\|\cdot\|$ and $g(x)=\langle$, for all $x \in U$. We knew before that $(U, \tau, g)$ is a Riemann-Wiener manifold. In this case we have the following:

$$
\begin{aligned}
d_{g}(x, y) & =|x-y| & & \text { if } x-y \in H \\
& =\infty & & \text { otherwise. }
\end{aligned}
$$

(3.3) is trivial by the convexity of $U$; (3.4) is a consequence of Example (i) of Theorem II.6 and Lemma III.8 (to appear later).

Proposition III.7. $d_{g}$ is an almost-metric on $\mathscr{W}$ in the following sense:

$$
d_{g}: \mathscr{W} \times \mathscr{W} \rightarrow[0, \infty]
$$

such that (1) $d_{g}(x, y)=0 \Leftrightarrow x=y$, (2) $d_{g}(x, y)=d_{g}(y, x)$ for all $x, y \in \mathscr{W},(3) d_{g}(x, z)$ $\leqq d_{g}(x, y)+d_{g}(y, z)$ for all $x, y, z \in \mathscr{W}$.

Proof. Obvious. Q.E.D.

Although $d_{g}$ is not a metric on $\mathscr{W}$, it is easy to see that, for each point $x$ in $\mathscr{W}$, $d_{g}$ is a metric on $R(x)$ and defines the original topology of $R(x)$. Here $R(x)$ is considered as the Riemannian manifold $(R(x), g)$ modelled on $H$. 
LEMMA III.8. Let $x$ be a fixed point in $\mathscr{W},(U, \varphi)$ a compatible chart at $x$ given by $\mathrm{RW}-3, z \in U$, then $z \in R(x) \Leftrightarrow d_{g}(z, x)<\infty$.

Proof. " $\Rightarrow$ " follows from the definition of $R(x)$ and its properties. Note that in this section, $U$ is taken to be connected. Therefore $R(x)$ is also connected.

" $\Leftarrow$ " Suppose $d_{g}(z, x)<\infty$, then it is easy to see that there exists a differentiable curve $r$ lying entirely in $U$ such that $r(0)=x, r(1)=z$ and $L_{g}(r)<\infty$. Since

$$
|\varphi \circ r(t)-\varphi(x)| \leqq \int_{0}^{t}\left|(\varphi \circ r)^{\prime}(s)\right| d s \leqq \max _{0 \leqq s \leqq t}\left\{\left\|\varphi_{r(s)}\right\|_{R_{r(s)}, H}\right\} L_{g}(r)<\infty
$$

it follows that $\varphi \circ r(t)-\varphi(x) \in H$. Hence $r(t) \in R(x)$, in particular $z=r(1) \in R(x)$. Q.E.D.

Proposition III.9. For any two points $x, y$ in $\mathscr{W}, d_{g}(x, y)<\infty$ if and only if there exist a finite number of points $z_{1}, \ldots, z_{n}$ in $\mathscr{W}$ such that $z_{1} \in R(x), z_{k+1} \in R\left(z_{k}\right)$, $k=1, \ldots, n-1, y \in R\left(z_{n}\right)$.

Proof. Apply the above lemma. Q.E.D.

(c) Proof of Theorem III.2. From Theorem III.5, it is sufficient to prove that $d_{g}(x, y)<\infty$ if and only if there exist charts $(U, \varphi),(V, \psi)$ at $x, y$ respectively such that $U \supset U(x), V \supset U(y)$ and $\psi(y)-\varphi(x) \in H$.

Sufficiency. By Remark (ii) of Theorem II.6, there exists a point $z \in U \cap V \cap R(x)$.

$$
z \in R(x) \Rightarrow d_{g}(z, x)<\infty \Rightarrow \varphi(z)-\varphi(x) \in H .
$$

Now $\psi(y)-\psi(z)=(\psi(y)-\varphi(x))+(\varphi(x)-\varphi(z))+(\varphi(z)-\psi(z))$. The first term is in $H$ by the assumption, the second term is in $H$ by (3.5), while the third term is in $H$ because $\varphi(z)-\psi(z)=\varphi \psi^{-1}(\psi(z))-\psi(z) \in H$ since $\varphi \psi^{-1}$ is admissible. Therefore $\psi(y)-\psi(z) \in H$, whence $z \in R(y)$ and it follows that $d_{g}(y, z)<\infty$. Finally

$$
d_{g}(x, y) \leqq d_{g}(x, z)+d_{g}(z, y)<\infty .
$$

Necessity. The existence of $(U, \varphi)$ and $(V, \psi)$ comes from (2.9). By Proposition III.9, there exists a finite number of points $z_{1}, z_{2}, \ldots, z_{n}$ such that $z_{1} \in R(x)$, $z_{k+1} \in R\left(z_{k}\right), k=1,2, \ldots, n-1$, and $z_{n} \in R(y)$. Let $\theta_{k}$ be a compatible chart at $z_{k}$, $k=1,2, \ldots, n$, then $\varphi\left(z_{1}\right)-\varphi(x) \in H$ and $\theta_{1}\left(z_{2}\right)-\theta_{1}\left(z_{1}\right) \in H$ since $z_{1} \in R(x)$ and $z_{2} \in R\left(z_{1}\right)$. Therefore

$$
\theta_{1}\left(z_{2}\right)-\varphi(x)=\left(\theta_{1}\left(z_{2}\right)-\theta_{1}\left(z_{1}\right)\right)+\left(\theta_{1}\left(z_{1}\right)-\varphi\left(z_{1}\right)\right)+\left(\varphi\left(z_{1}\right)-\varphi(x)\right)
$$

and $\theta_{1}\left(z_{2}\right)-\varphi(x) \in H$ by the same argument used in the Sufficiency part.

Similarly $\theta_{2}\left(z_{3}\right)-\varphi(x) \in H, \ldots, \theta_{n-1}\left(z_{n}\right)-\varphi(x) \in H, \theta_{n}(y)-\varphi(x) \in H$. Moreover, $\psi(y)-\theta_{n}(y) \in H$ since $\psi \circ \theta_{n}^{-1}$ is admissible. Hence

$$
\psi(y)-\varphi(x)=\left(\psi(y)-\theta_{n}(y)\right)+\left(\theta_{n}(y)-\varphi(x)\right) \in H .
$$

Q.E.D. 


\section{BIBLIOGRAPHY}

1. R. H. Cameron and W. T. Martin, Transformations of Wiener integrals under translations, Ann. of Math. (2) 45 (1944), 386-396. MR 6, 5.

2. - Transformation of Wiener integrals under a general class of linear transformations, Trans. Amer. Math. Soc. 58 (1945), 184-219. MR 7, 127.

3. - The transformation of Wiener integrals by nonlinear transformations, Trans. Amer. Math. Soc. 66 (1949), 253-283. MR 11, 116.

4. Ju. L. Daleckiǐ and Ja. I. Snaǐderman, Diffusion and quasi-invariant measures on infinitedimensional Lie groups, Funkcional. Anal. i Priložen. 3 (1969), no. 2, 88-90. (Russian) MR 40 \#2138.

5. I. M. Gel'fand and N. Ja. Vilenkin, Generalized functions. Vol. 4: Some applications of harmonic analysis, Fizmatgiz, Moscow, 1961; English transl., Academic Press, New York, 1964. MR 26 \#4173; MR 30 \#4152.

6. L. Gross, Classical analysis on a Hilbert space, Analysis in Function Space, M.I.T. Press, Cambridge, Mass., 1964, Chap. 4, pp. 51-68. MR 29 \#4423.

7. - Measurable functions on Hilbert space, Trans. Amer. Math. Soc. 105 (1962), 372-390. MR 26 \#5121.

8. — Abstract Wiener spaces, Proc. Fifth Berkeley Sympos. Math. Statist. and Probability (Berkeley, Calif., 1965/66), vol. 2, part 1: Contributions to Probability Theory, Univ. of California Press, Berkeley, Calif., 1967, pp. 31-42. MR 35 \#3027.

9. - Integration and nonlinear transformations in Hilbert space, Trans. Amer. Math. Soc. 94 (1960), 404-440. MR 22 \#2883.

10. — Potential theory on Hilbert space, J. Functional Analysis 1 (1967), 123-181. MR 37 \#3331.

11. I. M. Koval'chik, The Wiener integral, Uspehi Mat. Nauk 18 (1963), no. 1 (109), 97-134= Russian Math. Surveys 18 (1963), no. 1, 97-134. MR 36 \#5295.

12. S. Lang, Differentiable manifolds, Interscience, New York, 1966.

13. J. T. Schwartz, Nonlinear functional analysis, Gordon and Breach, New York, 1969.

14. I. E. Segal, Tensor algebras over Hilbert spaces. I, Trans. Amer. Math. Soc. 81 (1965), 106-134.

15. - Distributions in Hilbert space and canonical systems of operators, Trans. Amer. Math. Soc. 88 (1958), 12-41. MR 21 \#1545.

16. N. Wiener, The average value of a functional, Proc. London Math. Soc. (2) 22 (1923), 454-467.

CORNell University, IthACA, NeW YORK 14850 